\title{
17. Electrofisiología cardiaca
}

\subsection{Taquicardia ventricular sin pulso inducida por veneno de escorpión: un evento infrecuente}

\section{Radillo Alba Hugo Alberto}

Hospital Civil de Guadalajara «Fray Antonio Alcalde».

Tipo de estudio: Reporte de casos clínicos con revisión de fuentes

Introducción: Las zonas áridas del país son endémicas de distintas especies de escorpiones cuyos venenos son nocivos para ciertos mamíferos. Entre la presentación habitual del envenenamiento por escorpión figuran manifestaciones propias del sistema nervioso autónomo como la sialorrea, taquicardia y alteración del patrón respiratorio. En el presente caso reportamos un evento de muerte súbita abortada en una mujer joven. Descripción del caso: Mujer de 32 años, previamente saludable, sin factores de riesgo cardiovascular, hospitalizada por pérdida del estado de alerta, asociada con contracciones musculares involuntarias. Se inicia abordaje de síndrome convulsivo, pero se documenta parada cardiaca con trazo de taquicardia ventricular, remitida luego de desfibrilación. Durante los cuidados postparto se descarta alteración cardiaca estructural y se presencian múltiples eventos de taquicardia ventricular. Metodología: Se realizó una matriz de búsqueda intencionada con

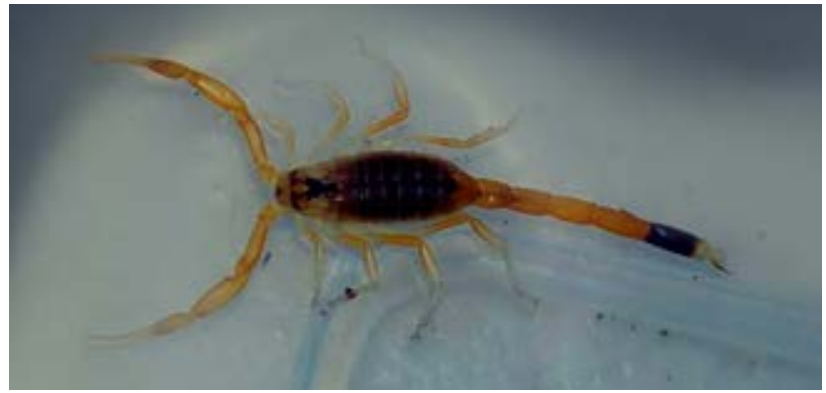

Figura 17.1.1.

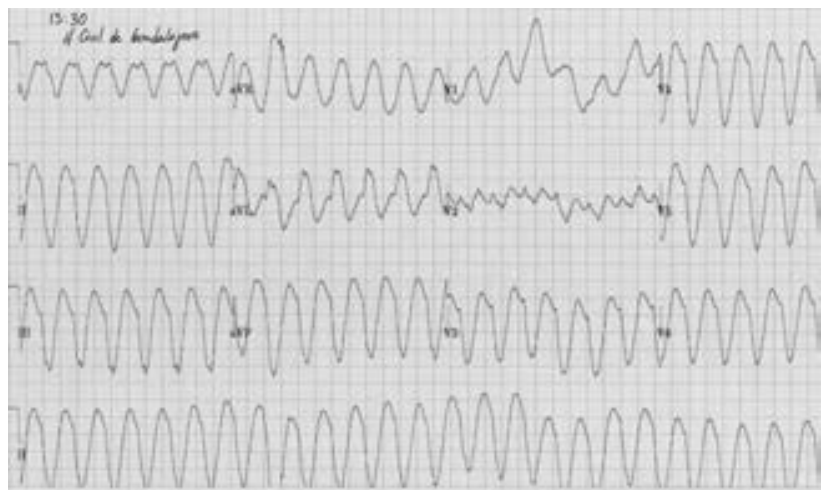

Figura 17.1.2. reportes de caso y recomendaciones de expertos en materia de arritmias inducidas por veneno de escorpión. Se reportan eventos esporádicos que impiden acuñar una prevalencia e incidencia exacta en países endémicos para especies nocivas de escorpión. Resultados: Al comparar con la bibliografía notamos la resonancia clínica y del contexto arrítmico de la paciente atendida en nuestro centro con aquellos atendidos en la India, Egipto y Siria. Si bien, las estrategias de urbanización hacen cada vez menos frecuente el envenenamiento por escorpión en comunidades mexicanas, no tenemos forma de predecir con exactitud el desenlace ominoso de los pacientes afectados. Discusión: Se puede abstraer del caso una presentación en extremo infrecuente, no sólo dado el hecho del rápido deterioro eléctrico de la paciente, sino por la réplica de eventos de taquicardia ventricular, mismos que se inhibieron a lo largo de la administración del antídoto hasta lograr la recuperación de la paciente, sin secuelas clínicamente observables.

\subsection{Disfunción de marcapaso por síndrome de reel}

Sánchez Vázquez Tomás, García Jiménez Yoloxóchitl, Hernández Bravo Mariana, Soto González Juan Ignacio IMSS UMAE No. 14 del Centro Médico Nacional «Adolfo Ruiz Cortines», Veracruz.

Tipo de estudio: Reporte de casos clínicos con revisión de fuentes

Introducción: El síndrome de reel se caracteriza por la rotación del generador del marcapasos sobre su eje transversal y el enrollamiento alrededor de éste de los electrodos, lo que ocasiona el desplazamiento de los mismos con la pérdida de la estimulación, esto causa disfunción del marcapasos. Su prevalencia es desconocida. Descripción del caso: Femenino de 79 años, hipertensa, con síndrome de bradicardiataquicardia e implante de marcapaso VVI en 2014. En 2019 presenta

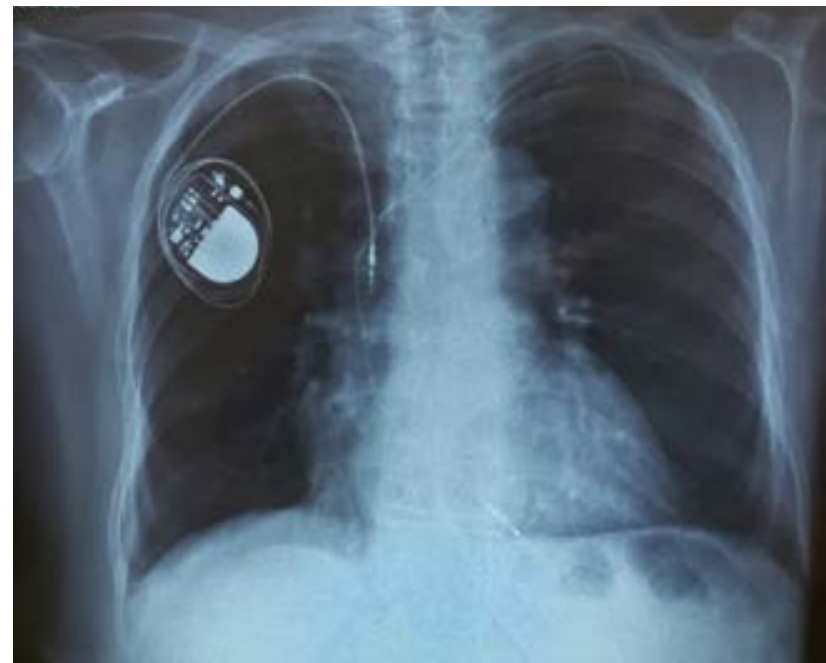

Figura 17.2.1. 
datos de bajo gasto y se documentan impedancias altas. Se implanta nuevo electrodo ventricular y generador en región subcutánea pectoral derecho, sin complicaciones. Dos semanas después de su egreso presenta palpitaciones y disfunción de captura y estimulación, con lo cual se diagnostica síndrome de reel. Metodología: Se realiza una revisión sistemática en revistas indexadas disponibles en la base de datos de PubMed-Medline con las palabras clave: síndrome de reel, complicaciones marcapaso, dando relevancia al factor de impacto. Se documentaron reportes de casos de esta patología. Resultados: La prevalencia del síndrome de reel se describe similar a la del síndrome de Twiddler de $0.07 \%$. Los factores asociados son la obesidad, sexo femenino, una bolsa del generador grande y ancianos. Nuestro caso tenía factores de riesgo para esta complicación. El seguimiento estrecho posterior al implante permite identificar estas complicaciones a largo plazo. Discusión: En la actualidad, las guías internacionales recomiendan un seguimiento periódico desde las primeras 72 horas del implante, en la segunda semana y a los 12 meses, continuando con intervalos de tres a 12 meses, sea este personal o remoto. El seguimiento clínico, radiográfico, electrocardiográfico y por telemetría,

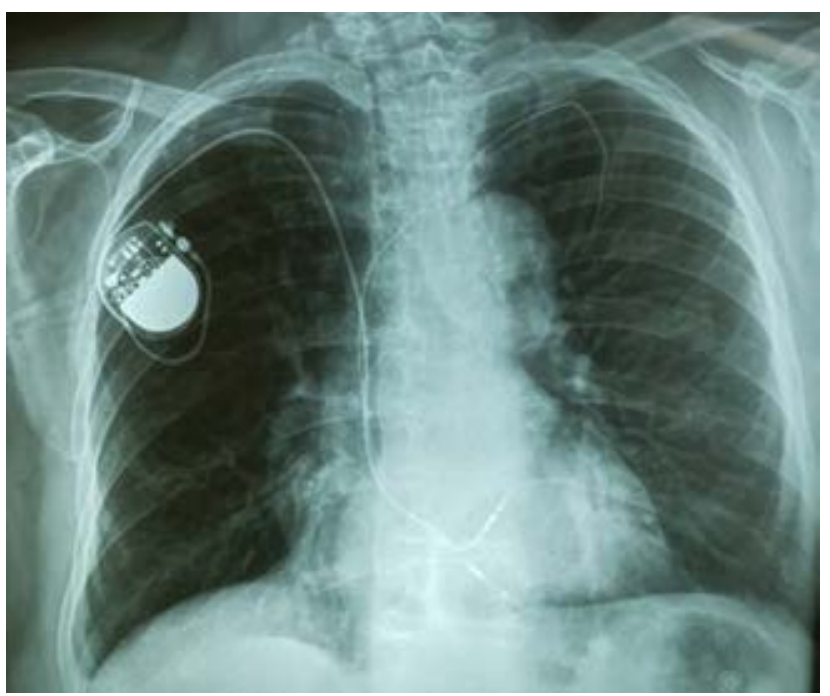

Figura 17.2.2. en especial en grupos de riesgo, nos permite identificar de manera oportuna estas complicaciones.

\subsection{Disincronía atrioventricular tardía en paciente adulto postoperado de cardiopatía congénita compleja cianótica}

Sánchez Rodríguez Roberto,* Arroyo Cristopher Germán, ${ }^{\ddagger}$

García Dávalos Israel, ${ }^{\ddagger}$ Salazar Lizárraga David, ${ }^{\ddagger}$ Márquez González Horacio, ${ }^{\ddagger}$

López Gallegos Diana, ${ }^{\ddagger}$ Yáñez Gutiérrez Lucelli ${ }^{\ddagger}$

* Instituto Mexicano del Seguro Social. * Hospital de Cardiología

del Centro Médico Nacional Siglo XXI, IMSS.

Tipo de estudio: Reporte de casos clínicos con revisión de fuentes

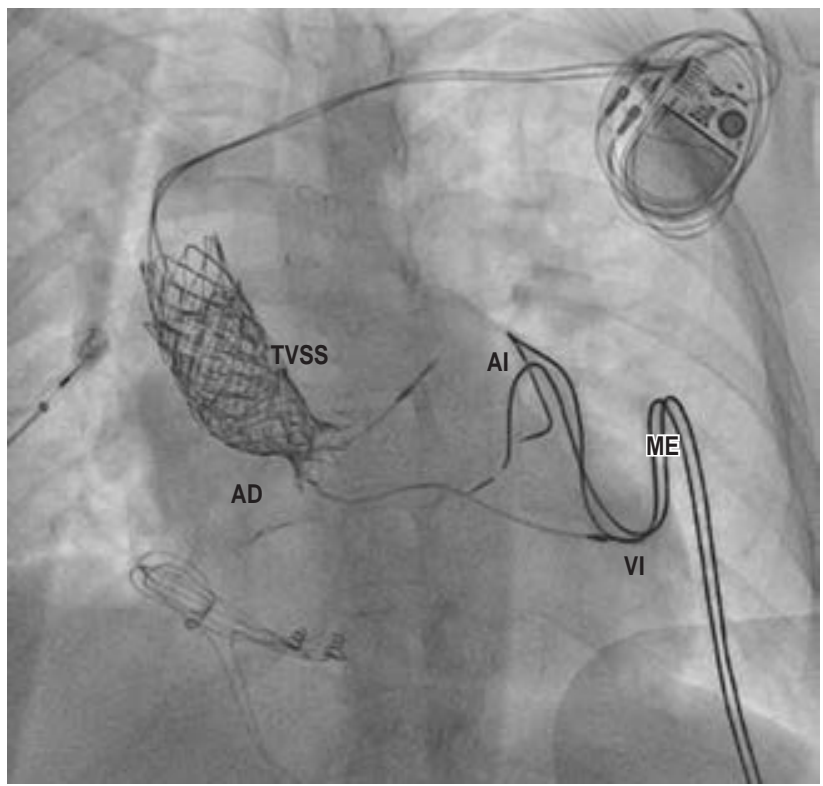

Figura 17.3.2: Se observa por fluoroscopia la implantación de marcapaso endocárdico bicameral, los cables de los electrodos pasan a través del túnel venoso sistémico superior (TVSS) y los stents dirigidos hacia la orejuela del atrio izquierdo (Al) y pared libre del ventrículo izquierdo (VI). $A D=$ aurícula derecha; $M E=$ marcapaso epicárdico.
Figura 17.3.1:

A) Radiografía de tórax con presencia de marcapaso epicárdico con hipertensión venocapilar grado II. B) Radiografía de tórax después de la implementación de marcapaso endocárdico bicameral,

los cables de electrodos pasan a través del túnel venoso sistémico superior (TVSS) y los stents hacia

la orejuela del atrio izquierdo

(Al). $A D$ = aurícula derecha;

$M E$ = marcapasos epicárdico;

$\mathrm{VI}=$ ventrículo izquierdo.
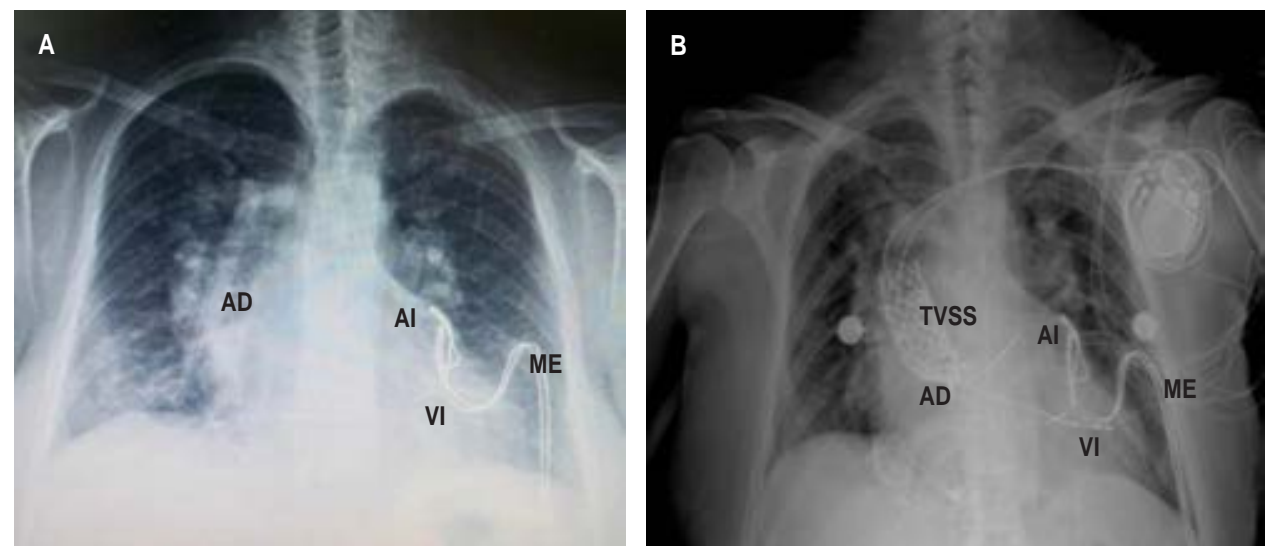
Introducción: La transposición de grandes arterias (TGA) es un defecto en el que los troncos arteriales son conectados a los ventrículos de morfología inapropiada. La supervivencia ha mejorado tras la introducción del switch atrial (fisiológico), descritos por Senning y Mustard, asociadas con secuelas tardías (especialmente arritmias, complicaciones relacionadas al túnel y disfunción ventricular derecha), por lo que en la actualidad se realiza cirugía de Jatene. Descripción del caso: Mujer de 52 años, antecedente de TGA corregida por Mustard a los dos años de vida, presentó bloqueo atrioventricular, por lo que requirió marcapaso epicárdico. Cursó un mes con disnea, secundario a disfunción del túnel, se realizó intervención y stents en el sitio de estenosis, con marcapaso disfuncional que ocasionó disincronía atrioventricular, se colocó marcapaso definitivo endocárdico a través del túnel. Metodología: A través del método booleano se realizó búsqueda de términos $\mathrm{MeSH}$ «Mustard» [and] «Transposition of the Great Arteries» en las bases de datos con repositorios de evidencia científica arbitraria indizada (PubMed) y no indizadas (Google Scholar). Resultados: La supervivencia de Mustard es de $80 \%$ después de los 25 años. La disfunción ventricular sistémica de inicio tardío es una complicación mayor asociada con el uso de marcapaso univentricular en pacientes con transposición de grandes arterias. El marcapaso biventricular previene el desarrollo de disincronía ventricular y posterior insuficiencia cardiaca de inicio tardío. Discusión: Paciente con supervivencia mayor de 40 años después de la corrección tipo Mustard, se asocia con excelentes resultados en las primeras dos décadas; sin embargo, el desarrollo de disfunción ventricular sistémica tardío sigue siendo un problema significativo. Quienes desarrollaron bloqueo atrioventricular deben ser sometidos a marcapaso biventricular para prevenir la disincronía atrioventricular que puede llegar a ocasionar daño a ventrículo sistémico. 